\title{
How COVID-19 interacts with interstitial lung disease
}

\author{
Katherine J. Myall, Jennifer L. Martinovic and Alex West
}

Dept of Respiratory Medicine, Guys' and St Thomas' NHS Foundation Trust, London, UK.

Corresponding author: Katherine J. Myall (Katherine.Myall@gstt.nhs.uk)

Check for
updates
Copyright OERS 2022
Breathe articles are open access
and distributed under the terms of
the Creative Commons Attribution
Non-Commercial Licence 4.0.

Received: 9 Dec 2021

Accepted: 18 Jan 2022
Shareable abstract (@ERSpublications)

COVID-19 has had profound effects on patients with pre-existing interstitial lung disease, and there is growing interest in the effect on the lung parenchyma in patients recovering from acute infection. https://bit.ly/33M5s4x

Cite this article as: Myall KJ, Martinovic JL, West A. How COVID-19 interacts with interstitial lung disease. Breathe 2022; 18: 210158 [DOI: 10.1183/20734735.0158-2021].

\section{Abstract}

The global pandemic caused by severe acute respiratory syndrome coronavirus 2 (SARS-CoV-2) has had far-reaching impacts on patients with interstitial lung disease (ILD), from diagnosis to management. In addition, after infection, persistent parenchymal change is associated with ongoing symptoms and functional impairment even in patients without pre-existing lung disease. The challenge of investigating and treating these patients has often fallen to ILD physicians. This review therefore seeks to explore the relationship between COVID-19 and the interstitium, as well as the model of care for patients with preexisting ILD and those patients with persistent disease following recovery from their initial infection.

\section{Educational aims}

- To understand the impact of the COVID-19 pandemic on patients with existing interstitial lung disease.

- To explore the development of interstitial lung disease after COVID-19 infection.

\section{Introduction}

From its first identification at the very end of 2019, through to its declaration as a Public Health Emergency of International Concern (PHEIC) on 30 January 2020 and later a pandemic on 11 March 2020, COVID-19 - the clinical disease caused by severe acute respiratory syndrome coronavirus 2 (SARS-CoV-2) - has been the cause of 235 million cases and 4.8 million deaths worldwide. This has impacted on the interstitial lung disease (ILD) community in numerous ways.

ILD describes a diverse group of diseases typified by excess deposition of collagen within the extracellular matrix of the lung parenchyma. This leads to restrictive lung disease and impairment of gas exchange and results in progressive dyspnoea, along with cough, fatigue and reduced life expectancy in many cases. There are over 200 different ILDs, including idiopathic pulmonary fibrosis (IPF), as well as those associated with autoimmune disease, or related to exposure to allergens.

This review seeks to evaluate the current published evidence in this field. We will consider the bidirectional effects of existing ILD on the course of COVID-19 in an individual, as well as the ILD which is now increasingly being described as an aftermath of COVID-19 infection. We will also consider the effects, both positive and negative, on the model of care for ILD patients during the pandemic, and the impact of lockdowns on patients with ILD. Given the scale of the pandemic, and as more people recover from more severe disease, the burden of affected patients, and demand for ILD services is only likely to increase.

\section{Effect of the COVID-19 pandemic on patients with existing ILD}

The pandemic has clearly had profound effects on the whole population. However, those with a pre-existing ILD have been peculiarly affected. It was assumed early in the pandemic that patients with 
ILD would be particularly vulnerable to the effects of COVID-19, as they have limited respiratory reserve, and in many cases are treated with medications which render them immunosuppressed. As a result, both patients and healthcare professionals were anxious to reduce the risk of infection as far as possible.

\section{Delivery of care to patients with ILD during the pandemic}

In addition to the more general effects of the pandemic on patients with ILD, there has been an enormous shift in the delivery of care during the COVID-19 pandemic. Health systems have been placed under unprecedented pressure by the pandemic, and the delivery of outpatient care has had to take place alongside the management of inpatients with COVID-19.

In order to diagnose an ILD, specialist centres use a multidisciplinary team (MDT) approach, combining clinical information with radiological and histopathological data. This would typically involve a face-to-face assessment for history and physical examination, blood tests, computed tomography (CT) scan of the chest and pulmonary function testing, with the possible addition of bronchoscopy or surgical lung biopsy. Following this, an MDT meeting is convened to confirm the diagnosis, and construct an appropriate treatment and therapy plan. Each facet of this approach has had to adapt to the pressures of the pandemic.

\section{Consultations}

The overriding aim during the COVID-19 pandemic has been to minimise unnecessary contact, but also to avoid delays in diagnosis and/or therapy. To this end, there has been a shift towards virtual consultation, either by telephone or video consultation in place of face-to-face assessment. This has the clear benefit of avoiding travel and exposure of the patient to infection. It is also generally acceptable to patients with chronic respiratory disease [1]. However, it does not allow for physical assessment of the patient, which might introduce a delay in diagnosis of disease or complication whilst awaiting this.

\section{Diagnostic tests}

Patients require diagnostic tests for a diagnosis of ILD to be made. During the pandemic, the increased burden on all healthcare services meant more difficulty in obtaining blood tests and CT scans, introducing a potential delay to diagnosis in some cases.

Pulmonary function testing presents a risk of spreading infection by aerosolising respiratory pathogens, such as SARS-CoV-2, and therefore access is much more limited than previously. This has meant reduced access for patients, and whilst home spirometry has been shown to be effective as a tool for monitoring progression in ILD [2], and offers a potential mechanism for remotely monitoring patients, provision of this service is certainly not universal. This has wide-reaching implications, as in many cases, treatment decisions may be based on either baseline or changing spirometric values.

Other diagnostic tests, including bronchoscopy and surgical lung biopsy, may also contribute to diagnosis. However, during the pandemic, exposure of the patient to the risk of potential infection whilst undergoing a procedure in the hospital is added to the risk of the procedure itself. This might alter the decision-making of the MDT, and in patients with a provisional diagnosis (50-90\% certainty), a period of observation or empiric treatment might be appropriate rather than pursuing these investigations [3].

In order to reduce unnecessary contact between clinicians, MDT meetings have also shifted online.

\section{Therapeutics}

The treatment of ILD will depend on the diagnosis made by the MDT and includes the use of immunosuppressive agents as well as antifibrotic therapy alongside pulmonary rehabilitation, oxygen therapy and the management of comorbid disease.

Immunosuppression for example with prednisolone, mycophenolate, cyclophosphamide or rituximab is common practice in the treatment of many forms of ILD. This led to concern that these patients might be particularly susceptible to severe infection. Data on this remains somewhat limited, although a meta-analysis did suggest there was an increased risk of severe disease amongst patients taking immunosuppression when compared with matched patients who were not (OR 3.29, 95\% CI 0.89-12.21, $\mathrm{p}=0.07$ ) [4]. This result did not reach statistical significance and was not specific to patients being treated for ILD [4]. Therefore, the risk of immunosuppression must be weighed against the risk of untreated ILD. Further consideration needs to be given to the timing of vaccination in regards to immunosuppression, as evidence suggests an impaired humoral response to available COVID-19 vaccines in patients receiving immunosuppression. It is often therefore appropriate to delay starting therapy until vaccination has taken place, and patients on immunosuppression should also have a third primary dose of the vaccine. 
There is no evidence that either of the currently available antifibrotics, pirfenidone and nintedanib, have any effect on the susceptibility of patients to COVID-19, or on disease severity. Because many countries require pulmonary function testing to initiate and monitor therapy, there has been a concern regarding delays to starting therapy. However, in many places, the requirements have been suspended or relaxed for a time to facilitate access to treatment.

Both immunosuppressants and antifibrotic medications require regular monitoring using blood tests. During the pandemic, patients and healthcare providers were understandably reluctant for this to occur in hospital, and this has led to the development of shared-care arrangements between primary and secondary care. Where this has been effective, for many patients, this has been extremely positive and is likely to continue.

\section{Oxygen therapy}

Patients with ILD should undergo regular assessment of their oxygen requirements, a process which was again interrupted by conversion to virtual clinic appointments. In addition, many UK authorities discontinued the use of ambulatory oxygen therapy, which has been shown to be beneficial for patients with ILD, because of concern over oxygen shortages [5]. However, patients already established on oxygen therapy tended to increase their use, possibly due to increased dyspnoea [6].

\section{Pulmonary rehabilitation}

Pulmonary rehabilitation remains a mainstay of therapy for ILD patients, and access was not available during the pandemic, compounding the lack of physical activity. In some areas, virtual pulmonary rehabilitation has been established, with overall positive patient experiences reported but with barriers being access to technology, and difficulty in creating personalised programmes for patients [7]. With many patients having become deconditioned during the pandemic, now that pulmonary rehabilitation is able to restart, a backlog of patients is expected.

In most countries, restrictions on movement were imposed in order to reduce community transmission of COVID-19. In the UK, patients who were thought to be clinically extremely vulnerable, including those with ILD, were asked to remain at home for a period of 12 weeks in spring 2020. Even though the restrictions have gradually lessened since this time, many patients have continued to exercise caution.

The effects of lockdown are multifaceted and may be particularly difficult for patients with ILD. In one study $57 \%$ of patients with ILD reported a reduction in their physical activity during lockdown, whereas in a large population-based study 64\% maintained normal exercise levels [6, 8]. Muscle deconditioning in patients with ILD is likely to exacerbate exercise intolerance and dyspnoea, the cardinal symptom of ILD. This is compounded by psychological factors, with patients with chronic diseases experiencing greater stress relating to lockdown than the general population in a questionnaire-based study in Northern Spain $(t(1991)=2.36, p=0.018$, dcohen=0.13) [9], with similar results seen for anxiety and depression. This effect increased with greater lengths of time spent in isolation $[9,10]$.

\section{COVID-19 infection in patients with existing ILD}

Patients with ILD have multiple factors which might predispose them to COVID-19 infection, and to more severe disease or death following infection. They have impaired lung function and may also be taking immunosuppressive medication, which may also affect response to vaccination. Acute exacerbation in response to other viral pathogens is common.

It appears that patients with ILD are indeed more susceptible to contracting COVID-19. In a Korean study of patients with COVID-19 compared with age, sex and location matched controls, LeE et al. [11] found that $0.8 \%$ of COVID-19 patients had an ILD diagnosis versus $0.4 \%$ of matched controls giving an adjusted OR for ILD of 2.02 (95\% CI 1.54-2.61; $\mathrm{p}<0.001)$.

LEE et al. [11] also demonstrated that patients with ILD in the COVID-19 cohort were more likely to have severe disease compared with those without (49.3\% versus $13.1 \%$, adjusted OR 2.32, 95\% CI 1.24-4.01) and patients with ILD are also more likely to succumb to infection. In a study of 46 ILD patients who developed COVID-19 infection, Esposito et al. [12] found that the odds ratio for death was 3.2 (95\% CI 1.3-7.3; $\mathrm{p}=0.01$ ) when compared to age- and sex-matched controls, and this increased to 4.3 (95\% CI 1.4-4.3; $\mathrm{p}=0.01$ ) after adjustment for age, sex, race, smoking status, cardiovascular disease (congestive heart failure and/or coronary artery disease), and any chronic immunosuppression. In a study of 28 ILD patients in Wuhan, China, HuANg et al. [13] found that patients with ILD requiring inpatient admission for 
COVID-19 infection were more likely to have cough, sputum and dyspnoea as presenting symptoms than COVID-19 patients without ILD. They also had increases in biochemical markers of disease severity.

Disease subtype appears to be important in disease susceptibility. In the study by LEE et al. [11], patients with all subtypes of ILD examined demonstrated increased frequency of infection, with the exception of chronic hypersensitivity pneumonitis, and the effect was most pronounced in patients with IPF. In the study by Esposito et al. [12], usual interstitial pneumonitis pattern (typically seen in IPF) was more common in non-survivors, but this did not reach statistical significance. Interestingly, the MUC5B promoter rs35705950T allele which is implicated in IPF associates with COVID-19, but appears to be protective against severe disease. This effect may be because MUC5B forms part of the innate immune response. In addition to this, short telomere length, which has been implicated in the development of both familial and sporadic IPF, associated both with COVID-19 infection and with more severe disease (defined by intensive care unit (ICU) admission or death without ICU) in a cohort of 70 inpatients [14].

As expected, more severe disease, as measured by pulmonary function testing, predicted poorer outcomes for patients with ILD. Drake et al. [15] found that forced vital capacity (FVC) $<80 \%$ was an independent risk factor for mortality (HR 1.72, 95\% CI 1.05-2.83), and in the study by Esposito et al. [12], the same effect was observed for transfer factor of the lung for carbon monoxide $\left(T_{\mathrm{LCO}}\right)$. In addition, the known risk factors for severe COVID-19, including age, male sex and obesity, are also risk factors for patients with ILD.

However, these patients were all confirmed cases who had been symptomatic at presentation. Patients with ILD commonly have cough and dyspnoea, so it is possible that this masked some cases of COVID-19. The true burden of COVID-19 amongst ILD patients is not known, as there are no studies including routine testing of patients with ILD. The cause of death for ILD patients is also not recorded in any of the mortality studies, although it is assumed to relate directly to COVID-19.

\section{Post-COVID ILD}

SARS-CoV-2 infection can cause a spectrum of disease from asymptomatic to fatal acute respiratory distress syndrome (ARDS). The most common manifestation, certainly in hospitalised patients, is COVID-19 pneumonia. Following COVID-19 pneumonia, several groups have described persistent parenchymal abnormalities which have been described as post-COVID ILD. The proportion of patients affected in survivors of COVID-19 varies between studies: from $72 \%$ in those mechanically ventilated [16]; to one third in those with a severe form of acute disease [17]; to 4.8\% of all hospital discharges [18]. Given the sheer number of patients infected with SARS-CoV-2 worldwide, and improving treatment in the acute phase meaning more survivors, the implications and future healthcare burden of this disease is staggering.

\section{Pathogenesis and histopathology}

Acutely, COVID-19 pneumonia causes radiological appearance of ground-glass opacification and consolidation [19]. Histopathologically this represents exudative diffuse alveolar damage, massive capillary congestion and oedema, organising pneumonia and the frequent superaddition of bacterial pneumonia. The diffuse alveolar damage, in particular, is very similar to that found in ARDS of other causes. More specific for COVID-19 is a high incidence of vascular pathology: microvascular injury, neutrophilic capillaritis, thrombogenic vasculopathy and microthrombi, and this is generally found despite anticoagulation treatment [20]. It should be noted that the majority of data relating to this comes from post-mortem specimens and may therefore not be reflective of the disease course in survivors. Transbronchial lung cryobiopsy has been carried out in patients with less advanced COVID-19 pneumonia and found a unique phenotypic pattern, different from both classical ILD and diffuse alveolar damage, but demonstrating the severe derangement of immune mechanisms triggered even early on in the viral infection [21].

The pathogenesis of fibrosis after COVID-19 is incompletely understood. Epithelial and endothelial injury occurs in the inflammatory phase of ARDS due to dysregulated release of matrix metalloproteinases, vascular endothelial growth factor, and the cytokines interleukin (IL)-6 and tumour necrosis factor- $\alpha$. This leads to Type II pneumocyte hyperplasia and the accumulation of fibroblasts and myofibroblasts and excessive deposition of collagen [22]. Pulmonary fibrosis is a recognised sequela of ARDS, but its clinical relevance has been questioned [23], and its incidence appeared to have been reducing in the era of lung protective ventilation [24]. A directly fibrotic toxic viral effect has therefore been postulated in addition. The spike protein of SARS-CoV-2 binds to the angiotensin converting enzyme (ACE)-II receptor found on epithelial cells in the respiratory tract (and elsewhere throughout the body) to gain entry to host cells. Binding of the ACE-II receptor upregulates the effects of angiotensin II, which is known to act as a 
powerful profibrotic [25]. The high rate of pulmonary vasculopathy and resultant hypoxic remodelling may also play a role.

\section{Radiology}

During early recovery ground-glass opacities (GGO) predominate radiologically. This may represent either ongoing inflammatory exudate in the alveoli, or early fine fibrosis. Over time, there is a reduction in GGO in favour of increasing reticulation and interlobular thickening, with slowing down of resolution of fibrotic change by day 49 [26]. A case series of 12 patients demonstrates changes in the same distribution as acute pneumonia at an average 92 days post-acute infection [27]. The radiological changes have been found to correlate with physiological impairment: $26 \%$ patients in the study conducted by HaN et al. [17] had abnormal $T_{\mathrm{LCO}}$ at 6 months, and this occurred more frequently in those with fibrotic-like changes than those without. McGroder et al. [16] found GGO, reticulations and traction bronchiectasis strongly correlated with a reduction in $T_{\mathrm{LCO}}$ (and less so with a reduction in FVC).

\section{Natural history of disease}

There remains considerable uncertainty as to whether these persistent fibrotic-like changes seen at 6-12 months will lead to eventual recovery, to fixed stable lung damage, or to have triggered a pathway of progressive fibrosis [25]. Myall et al. [18] demonstrated radiological and physiological improvement in a group of 30 patients found to have an organising pneumonia pattern on CT, after treatment with glucocorticoids. The natural progression of this pattern without steroid treatment has not been accurately described, but given the good response to corticosteroids in organising pneumonia of other post-infectious aetiology, a study including a comparator group is unlikely to be ethically justifiable.

Over the first 6-12 months it would appear the fibrotic-like changes seen post-COVID-19 follow a similar trajectory to those seen in the aftermath of other coronaviruses [28, 29]. So, perhaps comparison with previous zoonotic coronavirus outbreaks can provide some insight into the natural history of these fibrotic changes over a longer timeframe than is currently possible for COVID-19. The severe acute respiratory syndrome (SARS) outbreak centred on Southern China in 2002-2003 reported 8098 cases and 774 deaths and was caused by the genomically similar SARS-CoV, often now referred to as SARS-CoV-1. Several studies have investigated the long-term radiological and lung function outcomes in recovered SARS patients, over a period of up to 15 years. ZHANG et al. [30] followed up 71 SARS patients in an observational cohort study. Dividing the lung into radiological areas, they found an overall rate of $9.4 \%$ pulmonary lesions at enrolment, mainly GGO and strip-like consolidation. This rate reduced rapidly over the first year and then plateaued over the following 14 years. They found no substantial recovery in pulmonary function over this time. A 2016 study of 11 patients showed fibrotic-like changes remained present on high-resolution CT in 10 of the 11 patients up to 7 years after acute infection with SARS-CoV, although the extent improved over time. Intra- and interlobular septal thickening and fine reticulation increased their predominance over GGO over time [31].

\section{Risk factors}

Several features have been described conferring a higher risk of developing persistent interstitial changes in the early months after acute COVID-19 infection. Severity of initial illness appears to be the most closely linked to persistent CT abnormalities [17, 32, 33]. Age, ARDS, mechanical ventilation, male sex, serum lactate dehydrogenase, use of steroids and/or IL-6 blocker have all been described as conferring a greater risk of fibrosis in survivors [16, 17], although of course the degree to which any of these is a surrogate marker for disease severity is arguable. McGroder et al. [16] also found shorter blood leukocyte telomere length to be associated with higher risk of fibrotic-like change at 4 months.

\section{Treatment}

A search on ClinicalTrials.gov reveals 28 currently registered (October 2021) clinical trials investigating the effects of existing and novel therapeutics on the development or progression of fibrosis post-COVID-19.

The two antifibrotic agents currently licensed in the UK to treat IPF (nintedanib and pirfenidone) are under investigation in both the acute ARDS and post-acute phase of COVID-19 disease. These compounds have different mechanisms of action, but similar efficacy. Pirfenidone is a pyridine, with a downregulating effect on cytokines, although its exact mechanism of action as an antifibrotic is largely unknown. Nintedanib is a tyrosine kinase inhibitor. Both demonstrate around 50\% reduction in progression of fibrosis in IPF. The deuterated form of pirfenidone, deupirfenidone, is also under investigation. Nintedanib has also recently received licensing (but not National Institute for Health and Care Excellence approval for use in England) for progressive fibrotic ILD of other causes. Working on the basis that the virus is fibrogenic from early in the disease process, it stands to reason that the earlier these are introduced, the better the outcomes may be, 
although it must be appreciated that at best they will only block one facet of the complex immune and inflammatory dysregulation in acute COVID-19 [22]. Their use in the acute phase has been limited by concurrent hepatic enzyme derangement in this critically ill cohort (both agents are potentially hepatotoxic), and the high or universal rates of concurrent anticoagulation use during acute COVID-19 pneumonia which theoretically increases the bleeding risk associated with nintedanib. There are several double-blind, placebo-controlled, randomised controlled trials currently recruiting to assess the utility of these agents, but there are no currently published data relating to the safety or efficacy of these agents in this cohort.

Glucocorticoid treatment, namely prednisolone, has been used in an observational study on 30 patients with an ongoing radiological and functional deficit at 6 weeks. All 30 reported subjective improvement and had objective improvement in lung function/imaging [18]. It is assumed that this is treating an organising pneumonia component of interstitial disease and would be less likely to have any impact on established fibrosis.

Some traditional Chinese medicines are under study (tetrandine (ClinicalTrials.gov identifier: NCT04308317), fuzheng huayu and anluohuaxian) in placebo-controlled randomised controlled trials in China. Exciting novel approaches are also under study, including the MONACO cell therapy study (ClinicalTrials.gov identifier: NCT04805086), the IN01 vaccine, and the injection of autologous cellular stromal vascular fraction (cSVF). The outcomes following pulmonary rehabilitation are also under study; based on excellent outcomes from pulmonary rehabilitation in other areas of lung disease, this potentially offers another avenue to consider in the holistic management of these patients. Finally, lung transplant has been performed for post-COVID-19 fibrosis [34], but there is significant difficulty in selecting the right patients at the best time, given that the natural course of the disease remains unknown [30]. A review of currently available literature suggests waiting for 8 weeks without clinical improvement [35]; patients are often bridged with extracorporeal membrane oxygenation during this time [36]. The national statistics for cardiothoracic transplant in the UK in 2020-2021 have not yet been published so it is not known how many patients have undergone this procedure. In the USA 134 lung transplants for COVID-19 were recorded in 1 year (June 2020 to May 2021), 48 of whom were recorded as having post-COVID-19 fibrosis [37].

\section{Discussion}

It is clear that patients with ILD have been adversely affected by the pandemic at all stages of their diagnosis and treatment. Early symptoms of cough and dyspnoea may be incorrectly assumed to be related to COVID-19, leading to a delay in presentation. Following this, clinicians have had to balance the treatment of the overwhelming surge of patients with COVID-19 infection with delivering safe and effective diagnostics and care to patients with ILD. There has been limited access to diagnostic tests, and possible delays to treatment. In addition to this, along with the rest of the population, patients with ILD have been exposed to varying degrees of lockdown during the pandemic, with associated reduced physical activity, as well as psychological distress. Even as lockdowns are eased, patients with ILD feel particularly vulnerable given their lack of respiratory reserve, and in some cases anxiety relating to vaccine efficacy in the context of immunosuppression. Patients who have ILD appear to be more susceptible to COVID-19 infection and are at increased risk of severe disease and death when they do contract the disease.

Survivors of infection are left with radiological abnormalities, although debate remains as to whether this truly represents an ILD. Several groups have found persistent radiological change post-infection, with associated symptoms and physiological impairment.

Since more severe disease seems to predict the persistence of both symptoms and radiological abnormalities, and the pattern of ongoing change appears to relate to the regions of the lung involved in the acute infection, it may be that there is persistent inflammation in some cases. Radiological and histopathological evidence points towards a persistent organising pneumonia, and there is early evidence for glucocorticoids in such patients. There are some patients who do indeed seem to have CT features consistent with fibrosis, but Wells et al. [38], in an editorial in the same edition of Radiology as the study by HAN et al. [17], consider the difficulties in distinguishing between genuinely irreversible fibrosis, "fibrotic-like" changes, and non-fibrotic changes, and what this may mean in predicting the natural history of the disease. Clearly it is also possible in some patients that COVID-19 infection "unmasks" a previously undiagnosed ILD.

Given the huge numbers of patients affected worldwide, it behoves the ILD community to commit to further study of the effects of this novel pathogen on ILD patients, as well as the natural history of post-COVID ILD and its treatment. 
Conflict of interest: The authors have nothing to disclose.

\section{References}

1 Portnoy J, Waller M, Elliott T. Telemedicine in the era of COVID-19. J Allergy Clin Immunol 2020; 8: 1489-1491.

2 Russell AM, Adamali H, Molyneaux PL, et al. Daily home spirometry: an effective tool for detecting progression in idiopathic pulmonary fibrosis. Am J Respir Crit Care Med 2016; 194: 989-997.

3 Wong AW, Fidler L, Marcoux V, et al. Practical considerations for the diagnosis and treatment of fibrotic interstitial lung disease during the coronavirus disease 2019 pandemic. Chest 2020; 158: 1069-1078.

4 Gao Y, Chen Y, Liu M, et al. Impacts of immunosuppression and immunodeficiency on COVID-19: a systematic review and meta-analysis. J Infect 2020; 81: e93-e95.

5 Visca D, Mori L, Tsipouri V, et al. Effect of ambulatory oxygen on quality of life for patients with fibrotic lung disease (AmbOx): a prospective, open-label, mixed-method, crossover randomised controlled trial. Lancet Respir Med 2018; 6: 759-770.

6 Beltramo G, Cransac A, Favrolt N, et al. Impact of the COVID-19 lockdown on patients suffering from idiopathic interstitial pneumonia. Respir Med Res 2021; 79: 100808.

7 Lound A, de Luca BC, Kennedy ZA, et al. P81 The feasibility and acceptability of delivering virtual pulmonary rehabilitation during the COVID-19 pandemic. Thorax 2021; 76: Suppl. 1, A131.

8 Rogers NT, Waterlow NR, Brindle H, et al. Behavioral change towards reduced intensity physical activity is disproportionately prevalent among adults with serious health issues or self-perception of high risk during the UK COVID-19 lockdown. Front Public Health 2020; 8: 575091.

9 Ozamiz-Etxebarria N, Idoiaga Mondragon N, Dosil Santamaría M, et al. Psychological symptoms during the two stages of lockdown in response to the COVID-19 outbreak: an investigation in a sample of citizens in northern Spain. Front Psychol 2020; 11: 1491.

10 Louvardi M, Pelekasis P, Chrousos GP, et al. Mental health in chronic disease patients during the COVID-19 quarantine in Greece. Palliat Support Care 2020; 18: 394-399.

11 Lee $\mathrm{H}$, Choi H, Yang B, et al. Interstitial lung disease increases susceptibility to and severity of COVID-19. Eur Respir J 2021; 58: 2004125.

12 Esposito AJ, Menon AA, Ghosh AJ, et al. Increased odds of death for patients with interstitial lung disease and COVID-19: a case-control study. Am J Respir Crit Care Med 2020; 202: 1710-1713.

13 Huang $\mathrm{H}$, Zhang M, Chen C, et al. Clinical characteristics of COVID-19 in patients with preexisting ILD: A retrospective study in a single center in Wuhan, China. J Med Virol 2020; 92: 2742-2750.

14 Froidure A, Mahieu M, Hoton D, et al. Short telomeres increase the risk of COVID-19. Ageing (Albany NY) 2020; 12: 19911-19922.

15 Drake TM, Docherty AB, Harrison EM, et al. Outcome of hospitalization for COVID-19 in patients with interstitial lung disease an international multicenter study. Am J Respir Crit Care Med 2020; 202: 1656-1665.

16 McGroder CF, Zhang D, Choudhury MA, et al. Pulmonary fibrosis 4 months after COVID-19 is associated with severity of illness and blood leucocyte telomere length. Thorax 2021; 76: 1242-1245.

17 Han X, Fan Y, Alwalid O, et al. Six-month follow-up chest CT findings after severe COVID-19 pneumonia. Radiology 2021; 299: E177-E186.

18 Myall KJ, Mukherjee B, Castanheira AM, et al. Persistent post-COVID-19 interstitial lung disease. An observational study of corticosteroid treatment. Ann Am Thorac Soc 2021; 18: 799-806.

19 George PM, Barratt SL, Condliffe R, et al. Respiratory follow-up of patients with COVID-19 pneumonia. Thorax 2020; 75: 1009-1016.

20 Calabrese F, Pezzuto F, Fortarezza F, et al. Pulmonary pathology and COVID-19: lessons from autopsy. The experience of European Pulmonary Pathologists. Virchows Arch 2020; 477: 359-372.

21 Doglioni C, Ravaglia C, Chilosi M, et al. COVID-19 interstitial pneumonia: histological and immunohistochemical features on cryobiopsies. Respiration 2021; 100: 488-498.

22 George PM, Wells AU, Jenkins RG. Pulmonary fibrosis and COVID-19: the potential role for antifibrotic therapy. Lancet Respir Med 2020; 8: 807-815.

23 Wilcox ME, Patsios D, Murphy G, et al. Radiologic outcomes at 5 years after severe ARDS. Chest 2013; 143 920-926.

24 Burnham EL, Janssen WJ, Riches DWH, et al. The fibroproliferative response in acute respiratory distress syndrome: mechanisms and clinical significance. Eur Respir J 2014; 43: 276-285.

25 McDonald LT. Healing after COVID-19: are survivors at risk for pulmonary fibrosis? Am J Physiol Lung Cell Mol Physiol 2021; 320: 257-265.

26 Fang Y, Zhou J, Ding X, et al. Pulmonary fibrosis in critical ill patients recovered from COVID-19 pneumonia: preliminary experience. Am J Emerg Med 2020; 38: 2134-2138.

27 Gulati A, Lakhani P. Interstitial lung abnormalities and pulmonary fibrosis in COVID-19 patients: a short-term follow-up case series. Clin Imaging 2021; 77: 180-186.

28 Hui D. Impact of severe acute respiratory syndrome (SARS) on pulmonary function, functional capacity and quality of life in a cohort of survivors. Thorax 2005; 60: 401-409. 
$29 \mathrm{Ng}$ CK, Chan JWM, Kwan TL, et al. Six month radiological and physiological outcomes in severe acute respiratory syndrome (SARS) survivors. Thorax 2004; 59: 889-891.

30 Zhang $\mathrm{P}$, Li J, Liu $\mathrm{H}$, et al. Long-term bone and lung consequences associated with hospital-acquired severe acute respiratory syndrome: a 15-year follow-up from a prospective cohort study. Bone Res 2020; 8: 8.

31 Wu X, Dong D, Ma D. Thin-section computed tomography manifestations during convalescence and long-term follow-up of patients with severe acute respiratory syndrome (SARS). Med Sci Monit 2016; 22: 2793-2799.

32 Huang Y, Tan C, Wu J, et al. Impact of coronavirus disease 2019 on pulmonary function in early convalescence phase. Respir Res 2020; 21: 163.

33 Huang W, Wu Q, Chen Z, et al. The potential indicators for pulmonary fibrosis in survivors of severe COVID-19. J Infect 2021; 82: e5-e7.

34 Kurihara C, Bharat A. First successful lung transplantation for pulmonary fibrosis due to severe COVID-19 infection in the US. J Heart Lung Transplant 2021; 40: Suppl., S499-S500.

35 King CS, Mannem H, Kukreja J, et al. Lung transplantation for patients with COVID-19. Chest 2022; 161: 169-178.

36 Schaheen L, Bremner RM, Walia R, et al. Lung transplantation for coronavirus disease 2019 (COVID-19): The who, what, where, when, and why. J Thorac Cardiovasc Surg 2022; 163: 865-868.

37 Roach A, Chikwe J, Catarino $\mathrm{P}$, et al. Lung transplantation for Covid-19-related respiratory failure in the United States. N Engl J Med 2022; in press [https://doi.org/10.1056/NEJMc2117024].

38 Wells A, Deveraj A, Desai S. Interstitial lung disease after COVID-19 infection: a catalogue of uncertainties. Radiology 2021; 299: E216-E218. 